This is the final peer-reviewed accepted manuscript of:

R. Bolt, M. Magno, T. Burger, A. Romani and L. Benini

Kinetic AC/DC Converter for Electromagnetic Energy Harvesting in Autonomous Wearable Devices

in

IEEE Transactions on Circuits and Systems II: Express Briefs, vol. 64, no. 12, pp. 1422-1426, Dec. 2017

The final published version is available online at:

https://doi.org/10.1002/ppap.201500143

Rights / License:

The terms and conditions for the reuse of this version of the manuscript are specified in the publishing policy. For all terms of use and more information see the publisher's website

This item was downloaded from IRIS Università di Bologna (https://cris.unibo.it/)

When citing, please refer to the published version. 


\title{
Kinetic AC/DC Converter for Electromagnetic Energy Harvesting in Autonomous Wearable Devices
}

\author{
Robin Bolt*, Michele Magno*o, Thomas Burger*, Aldo Romani ${ }^{\circ}$, Luca Benini*o \\ *Dept. of Information Technology and Electrical Engineering, ETH Zurich, Switzerland \\ ${ }^{\circ}$ Dept. of Electrical, Electronic, and Information Engineering, University of Bologna, Italy
}

\begin{abstract}
A nano-power integrated circuit is designed to convert the AC output of an electromagnetic micro generator for wearable kinetic energy harvesting to DC usable power. The converter is designed to minimize the quiescent current and to maximize the energy conversion and maximum power point tracking efficiency from the kinetic energy harvester. The architecture implements an AC/DC chopper exploiting the intrinsic inductance of the transducer combined with a zero-drop rectifier. An optimal switching scheme is derived for the converter and a corresponding control circuit is developed and optimized for low power consumption. The converter is designed in a SMIC 130nm process, and circuit simulations show an efficiency of 87 $\mathbf{8 8 \%}$ with respect to the maximum power point of the transducer with a battery supply voltage ranging from $3.5 \mathrm{~V}$ to $4.2 \mathrm{~V}$. The quiescent current of the converter is as low as $250 \mathrm{nA}$.
\end{abstract}

Index Terms-Wearable devices, Energy Harvesting, Kinetic Energy Harvesting, Low Power Design, AC-DC Converter

\section{INTRODUCTION}

$I^{n}$ n recent years, wearable technologies incorporating sensors have increasingly entered our lives. Examples include people wearing "smart" objects, from clothing to phones, glasses and watches, which in combination achieve smart homes, healthcare and lifestyles. Many forecasts are claiming that the trend toward billions of intelligent and connected devices will herald the era of the Internet of Things (IoT). A key IoT challenge is the need to efficiently supply energy to enddevices, and especially for wearable devices this is mostly done using batteries. Unfortunately, batteries store limited amount of energy, and a suitable trade-off between capacity, mass, and volume has to be established. The problem is exacerbated by the constant desire of application developers to provide more functionalities in wearable devices.

Energy Harvesting (EH), broadly defined, is a promising set of technologies to overcome the limitation of short lifetime provided by contemporary battery technologies [1]-[4]. In the last decade, EH technologies have matured, finding use in many application scenarios such as smart grid and wireless sensor networks [1]. In fact, many companies are already selling multisource EH systems for commercial and industrial environments [1]. Recently, advances have been made in miniaturizing EH devices to supply wearable devices by exploiting ambient energy in the form of motion, thermal gradients, light and electromagnetic radiation. However, strict form factor and weight constraints make wearable energy harvesting a challenging target. The most promising energy sources for wearable devices are light and motion. For this reason, many researchers invest considerable effort to design small form factor, flexible solar panels and kinetic energy harvesters [1][5]
Today, solar harvesting can generate power in the $\mathrm{mW}$ range for wearable devices under sufficient amounts of light. This limits wearable applications, considering that most parts of the body are covered with clothing. Kinetic energy harvesters also have issues, most of them do not perform well enough with uneven power delivery coming from varying frequency components resulting from unpredictable movements of the human body[1]. In this context, using a mechanical energy buffer [6] decouples the output of the generator from the input motion, so that the generated signals occurs with an almost constant frequency, which drastically improves the efficiency of the system. Mechanically buffered kinetic harvesters have been investigated and improved since Seiko presented the Automatic Generating System (AGS). A novel device is the MSG 26.4 from Kinetron [7] that uses a mechanical spring as an intermittent energy buffer to drive the electrical generator.

In fully autonomous systems that rely on energy harvesting as their own energy source, energy availability is the main performance constraint [8][9]. Therefore, increasing the efficiency of the harvester circuit is a key goal. Usually kinetic energy harvesting generates AC power that needs to be converted to DC to be used by wearable devices[8]-[10]. Converters are a critical block in determining the conversion efficiency of a harvester, which is defined as the power that effectively flows to the energy storage over the maximum available power at the source. To improve the efficiency, losses must be minimized [11]. In addition, an accurate maximum power point (MPP) tracking is also of utmost importance[1].

In this work, we design a highly efficient power converter for wearable kinetic energy harvesting that uses an AC/DC chopper combined with a zero voltage drop Negative Voltage Converter (NVC). The NVC consists of rectifying cross-coupled MOSFETs connected in a bridge configuration, which offer negligible voltage drop when enabled. In [11], it was shown that NVC based rectifiers achieve a higher conversion efficiency from input to output (of over 90\%) due to the very low ONresistance of the cross-coupled MOSFETs. Since the internal inductance of the generator is exploited during power conversion, no external magnetics are required. However, there is still the need of biasing the input sources as close as possible to their MPP. As a novel feature of the proposed solution, the internal inductance of the kinetic generator is also exploited to maximize both the MPP tracking accuracy and the overall conversion efficiency. Thus, the main contribution of this paper is the design of a novel architecture, along with the design of an IC implementation and the presentation of results based on simulation, obtained using in-field measurements and characterization of a commercial kinetic energy harvester. 


\section{RELATED WORKS}

In recent years, wearable devices have received increasing attention from academic and industrial researchers. Today, there are many wearable products that are employed in the sport and health care domains [13]. However, achieving a selfpowered system through the use of energy harvesters (EH), which capture energy from the environment, combined with various storage devices such as batteries (commonly LiPo, rechargeable) is still challenging due to the limited form factor and weight [14]-[17]. Since wearable devices are expected to be mostly used on the human body, the most commonly available ambient energy sources are photovoltaic, thermal or kinetic energy [18], [19]. To maximize the energy harvested, there have been numerous recent studies in the field of converters [11]-[26][25], supporting the claim that high conversion efficiency associated with low quiescent current are necessary when the input power is limited as is the case of wearable devices. This is also an important feature of our approach that aims at maximizing the energy converted from the kinetic transducer while minimizing the quiescent consumption and losses.

As mentioned before, to convert kinetic energy into a usable DC energy, a low power and highly efficient AC/DC converter is needed. There are many previous works proposing solutions especially for piezoelectric transducers [27][28]. In particular, in [28] a nano-power integrated circuit optimized for piezoelectric energy harvesting was presented. The previous solution shows the benefits of using a NVC to maximize efficiency of the AC-DC converter. However, differently from our approach, the integrated circuit is required to synchronize with vibration peaks and thus undergoes a more complex control scheme. Another difference consists in the requirement of a relatively large external inductor.

Unlike power conversion from piezoelectric transducers, inductive output impedance has to be considered when dealing with electromagnetic energy harvesters. In [29], an impedance matching network was used to turn the inductive output impedance of an electromagnetic transducer into capacitive form, thus allowing the use of a more conventional piezoelectric harvesting circuit. In [21], a more sophisticated impedance matching approach based on bridgeless resonant AC/DC conversion was presented. However, the latter two works still require synchronization with input $\mathrm{AC}$ voltages, as well as the use of external inductors.

In [30] and [31] an integrated circuit converting power by means of an active full-bridge rectifier circuit from a compact electromagnetic (EM) generator has been presented. In [31] ideal diodes were emulated, which cancels all the associated power losses. Unfortunately, the circuit required a dual supply rail, which has to be generated by charge-pump circuits powered by a secondary coil. Moreover, although losses are minimized, no explicit MPP tracking (MPPT) is performed. An active full-wave rectifier was also adopted in [29], along with the design of an adaptive charge pump for MPPT purposes without external bulky inductors. In both cases, a NVC was used to interface the transducer. Also in this case, the losses associated with active rectification are extremely low. The main drawback of the charge pump is the relatively low conversion efficiency of approximately 50\%. In our solution, we still adopt a NVC rectifier, but our approach achieves a higher efficiency and MPPT accuracy by adopting an AC/DC chopping technique that exploits the internal inductance of the generator.

In general, although the proposed work adopts a widely used rectifying interface like the NVC, it explores a different power conversion topology and architecture. However, the main idea behind this work is to implement an AC/DC chopper combined with a zero voltage drop NVC exploiting the internal inductance of the kinetic generator, to improve conversion efficiency with a control circuit that maximizes the MPPT accuracy. More specifically, the control circuit determines the instantaneous current by sampling voltages on the impedance matching capacitor. As further design objectives, we targeted low losses and low intrinsic consumption of the circuits.

\section{KINETIC ENERGY HARVESTING}

In this paper, we present the design of a highly-efficient and low-quiescent-current AC-DC converter to convert the AC signal of the kinetic energy harvester to charge a $3.7 \mathrm{~V}$ battery.

Fig. 1 shows a high-level diagram of the kinetic energy harvesting system presented in this paper. The Kinetron MGS 26.4 is used as the kinetic generator, the designed integrated circuit includes a switching circuit combined with a NVC converter to maximize the conversion energy of the MGS 26.4. Thus, the idea explored in this work is to implement an AC/DC chopper, powered by the electromagnetic generator and exploiting the internal inductance of the generator. The switch $\mathrm{S}$ is controlled by a low-power control logic that senses the current on the capacitor $C$ that also matches the impedance of the transducer. This is accomplished by means of voltage-only measurements.

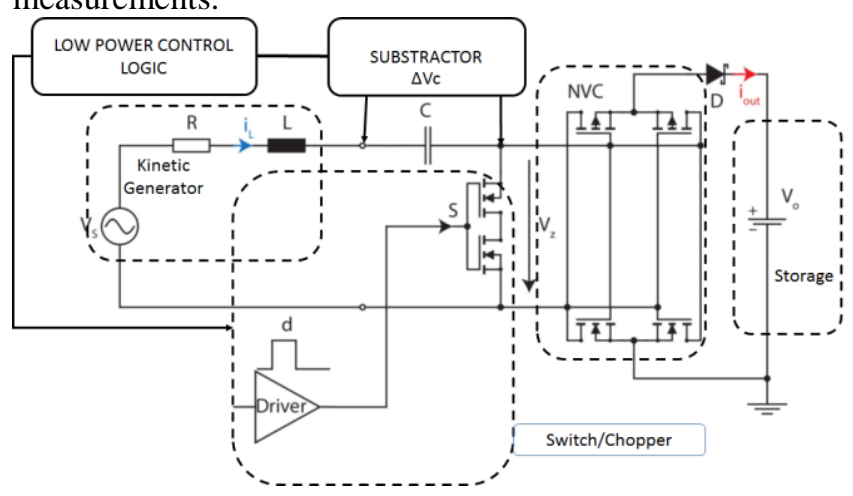

Fig. 1 Simplified Overview of the designed kinetic energy harvesting

\section{A. Kinetic Energy Generator}

The Micro generator system 26.4 (named because of its 26.4 $\mathrm{mm}$ diameter) from Kinetron is used in this work [7]. Tilting the device, an internal weight tenses a spring over mechanical rectifiers. A spring barrel attached to a mechanical rectifier then puts a certain amount of torque to its gear that drives a rotor of the electric generator. As motion happens, the barrel spring progressively winds. Since the spring unwinds when it reaches a certain amount of torque, the harvester generates an AC signal (burst) that is independent from the frequency of the motion of the oscillating weight Fig. 2. One full turn of the oscillating weight generates 4.25 bursts (signal pulses) of electrical energy.

The micro generator consists of a 14-pole resin bounded $\mathrm{Sm}_{2} \mathrm{Co}_{17}$ magnet and a claw pole stator that includes a 1140 turn 
winding coil with a resistance of $320 \mathrm{Ohm}$. One rotation of the magnet induces an AC voltage of 7 sinusoidal waves. The amplitude is proportional to the speed of the turning magnet. An average moving person produces 4'000 revolutions of the oscillating weight per day (when worn at the wrist). This results in approximately $17^{\prime} 000$ bursts per day. We measured an average energy of $43.8 \mu \mathrm{J}$ per energy burst. Hence, with about 17 '000 energy bursts a day, the harvester produces about $760 \mathrm{~mJ}$ per day, which is $27 \%$ higher than actually stated in the data sheet [7].

Fig. 2 shows the AC open circuit voltage output of the generator (a) and with a $1.33 \mathrm{k} \Omega$ load. The variations in the frequency are due to the non-constant angular velocity of the rotor. Since a complete rotation generates seven sinusoidal waves, a faster rotor speed will raise the frequency of the signal. As expected, a high-impedance load results in a longer and wider burst because of the lower dissipated power. Hence, the duration of a burst depends on the load (approximately $50 \mathrm{~ms}$ to $280 \mathrm{~ms}$ ). The amplitude of the generator goes up to $7 \mathrm{~V}$ (closed loop) and its frequency varies between $500 \mathrm{~Hz}$ and $2000 \mathrm{~Hz}$

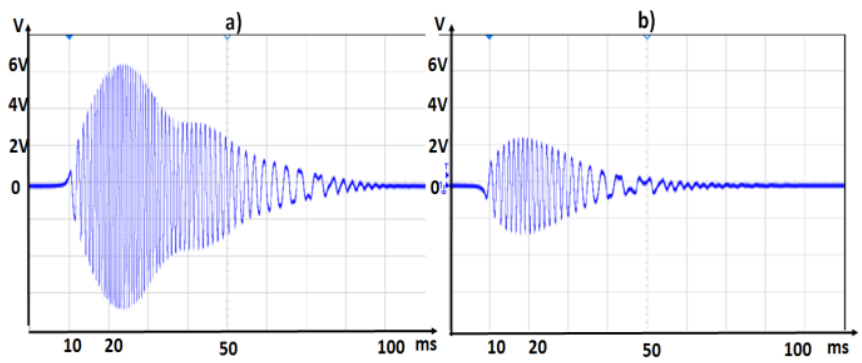

Fig. 2 (a) Measured open circuit waveform of a harvester burst, (b) waveform of a harvester burst with $1.33 \mathrm{kOhm}$ load

\section{B. AC-DC Converter}

To convert the AC output of MGS 26.4 output (Fig. 2) into a usable DC signal, an AC/DC converter is needed. We adopted a passive negative voltage converter (passive NVC) [28] that offers better conversion efficiency when compared with diode bridges. Using a NVC, composed of cross-connected MOSFETs, and a Schottky diode D preventing current backflow, allows a much smaller voltage drop than a diode bridge, due to the low on-resistance of the MOSFETs. However, in order to start conducting, the input voltage needs to exceed the threshold voltage of the MOSFETs, which can be higher than that of Schottky diodes. Then, although this was not implemented, using a Schottky diode bridge in parallel with the MOSFETs might therefore help to lower the voltage at which the circuit starts and stops conducting current.

The chopper switch is realized by two back-to-back series connected nMOS transistors. An additional series capacitor C is used for matching the imaginary part of the output impedance, in order to increase the power extracted from the transducer. The switch $\mathrm{S}$ is operated at a switching frequency $f_{\mathrm{sw}}=1 / T_{\mathrm{Sw}}$, and the turn-on time of $\mathrm{S}$ is given by duty cycle

$$
d=\frac{\text { Turn on time of } S}{\text { Switching period Tsw }}
$$

To get the optimal switching frequency, a simulation of the output power vs. switching frequency for fixed values of $d$ and $\mathrm{C}$ was performed, with the basic conversion circuit of Fig.3. The results in Fig. 4 show that a switching frequency $\mathrm{f}_{\mathrm{sw}}$ of approximately $30 \mathrm{kHz}$ maximizes the output power. In this work, $\mathrm{f}_{\mathrm{sw}}$ is fixed to $32 \mathrm{kHz}$ which is at least one order of magnitude higher than the maximum frequency of the harvester generator (MGS 26.4). Such frequency is a good compromise between sufficient oversampling such that the generator voltage remains about constant during inductor current build up and the switching losses that are proportional to $\mathrm{f}_{\text {sw.. }}$ In our design, the external pin, Ext clock in Fig. 5, provides the $32 \mathrm{kHz}$ clock from an external source.

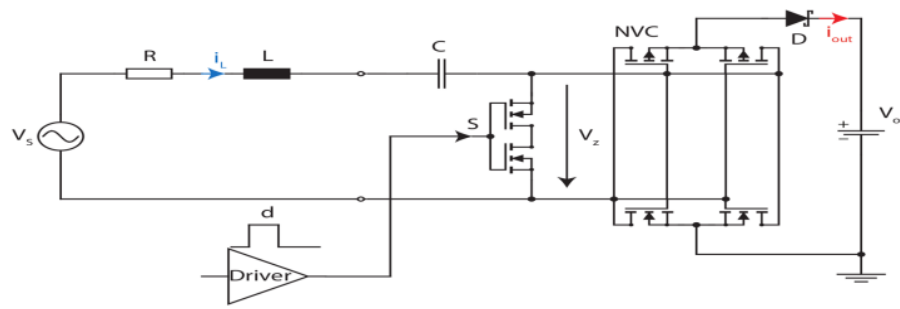

Fig. 3 Architecture of AC/DC converter designed in this work plus driver that sends the according switching signal to $\mathrm{S}, \mathrm{C}=400 \mathrm{nF}$, NMOS width of $1 \mathrm{~mm}$ and minimal length, $\mathrm{D}$ is Schottky diode BAT754

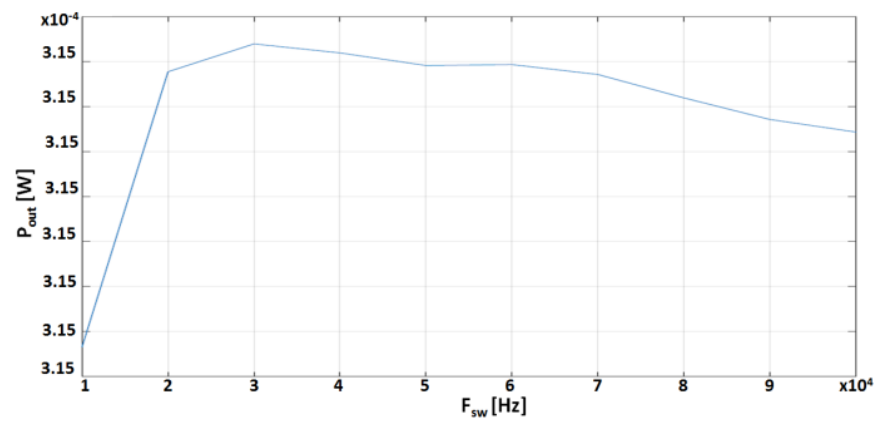

Fig. 4 Output power Pout as a function of $\mathrm{f}_{\mathrm{sw}}$ for a single burst, with $\mathrm{C}=400 \mathrm{nF}$; $\mathrm{d}=0.55$

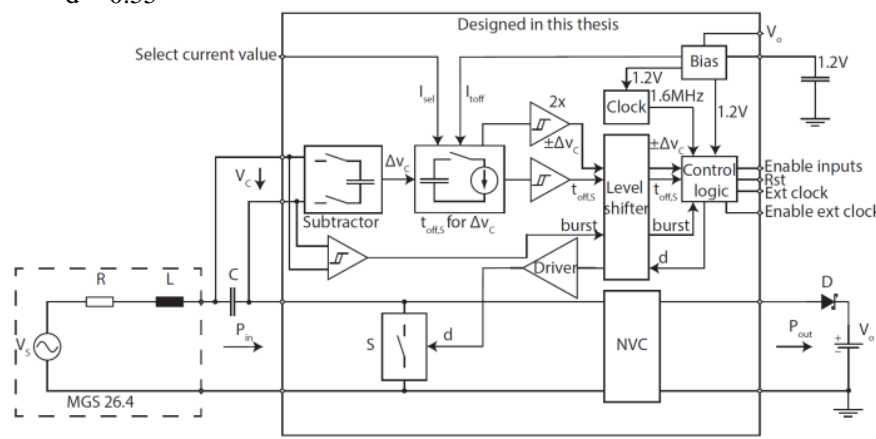

Fig. 5 Detailed overview of the designed circuits. The circuit recharges the battery Vo, which is also needed to generate the internal bias of the designed circuit.

\section{Control Circuitry}

Fig. 5 shows a detailed block diagram of the designed integrated circuit. The main building blocks in the control path are an input signal comparator, an input signal differentiator (subtractor) with a timing circuit, a control logic and a driver for the switch $\mathrm{S}$. In between bursts, only the input comparator, biased at $15 \mathrm{nA}$ in subthreshold region, the bias and the control logic are enabled to minimize power consumption. When a sufficiently 
high input voltage $\mathrm{V}_{\mathrm{c}}$ is detected, then the "burst"-signal activates the $1.6 \mathrm{MHz}$ clock over the controller, and the control path with differentiator and comparators is enabled, sampling the input $\mathrm{V}_{\mathrm{c}}$ at $32 \mathrm{kHz}$. When the burst dies off then the control path is switched off again with the same input comparator. The input and output circuits are designed with I/O-transistors tolerating the higher voltages on the generator and the battery side. Level shifters convert this power domain down to and up from a $1.2 \mathrm{~V}$ domain, supplied by an internal LDO, for the clock and control logic that can be realized with much lower power consumption at $1.2 \mathrm{~V}$.

\section{Switching Control Circuit}

In Fig. 3 the output current can be related to the inductor current by $\mathrm{i}_{\text {out }} \approx(1-\mathrm{d}) \times \mathrm{i}_{\mathrm{L}}$ like in the current calculation of a converter with similar topology (inductor $\mathrm{L}, \mathrm{S}, \mathrm{d}$ ). In order to obtain a measure of the generator inductor current the difference voltage $\Delta \mathrm{V}_{\mathrm{c}}$ between two clock cycles is derived with a switched capacitor circuit. Based on this measurement, the off-time $\left(\mathrm{t}_{\mathrm{off}}\right)$ of $\mathrm{S}$ that maximizes the conversion efficiency was determined. Fig. 6 shows the relationship.

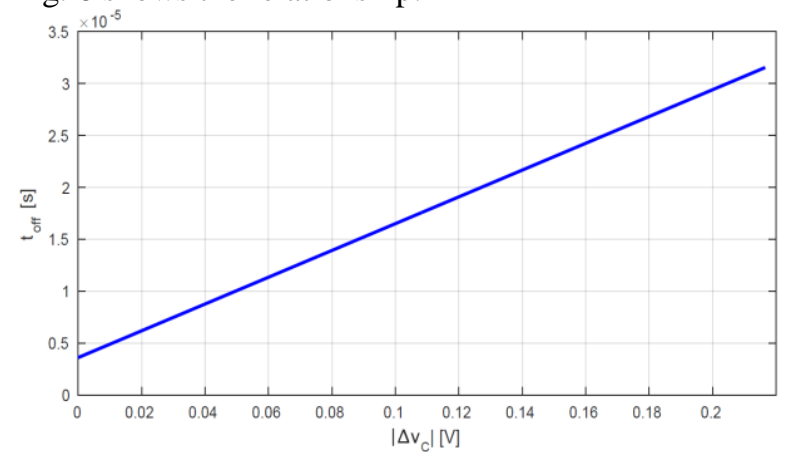

Fig. $6 \mathrm{t}_{\text {off }}$ related to the voltage difference over $\mathrm{C} \Delta \mathrm{V}_{\mathrm{c}}$ for $\mathrm{f}_{\mathrm{sw}}=32 \mathrm{kHz}, \mathrm{C}=$ $400 \mathrm{nF}$

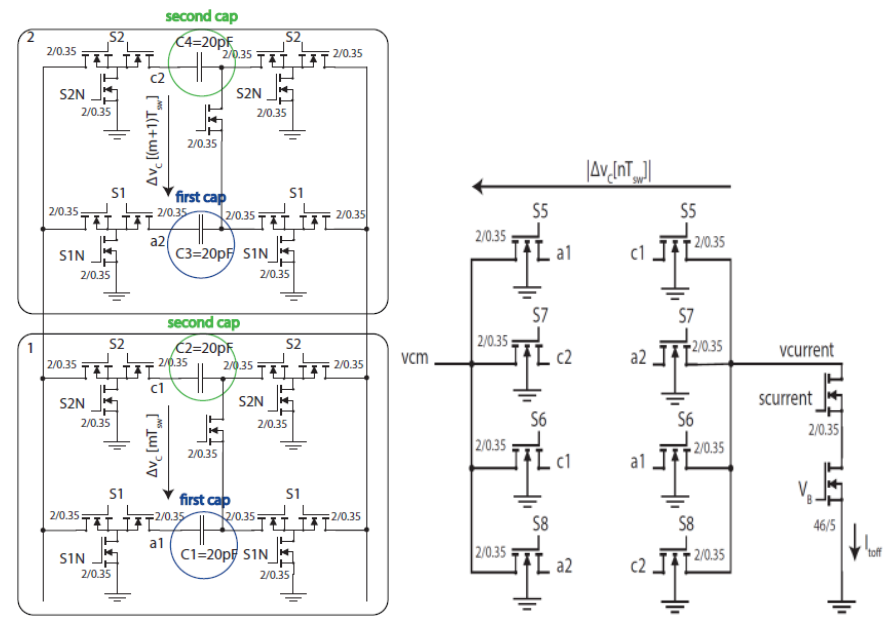

Fig. 7 Voltage subtraction circuit, $\mathrm{m}=1,3, .$. (left) Circuit for $\mathrm{t}_{\mathrm{off}} \propto \Delta \mathrm{Vc}, \mathrm{n}=1,2, .$. (right)

Fig. 7 shows the details of the designed subtraction circuit. In the subtraction circuit, as the first step, the voltage $V_{c}$ over $C$ is sampled on the two "first" capacitors. On the second switching cycle, the voltage $V_{c}$ is sampled on the "second" capacitors. The first and second cap are then connected together in box " 1 " shown in the left of Fig. 7 to obtain the voltage difference $\Delta \mathrm{Vc}\left[\mathrm{mT}_{\mathrm{sw}}\right]$ or in box " 2 " shown in the left of Fig. 7
$\Delta \mathrm{Vc}\left[(\mathrm{m}+1) \mathrm{T}_{\mathrm{sw}}\right]$, where $\mathrm{m}=1,3,5, \ldots . \Delta \mathrm{Vc}$ is then applied between the nodes 'vcm' and 'vcurrent' as indicated in Fig. 7 (right). The voltage ' $\mathrm{vcm}$ ' is added in order to set the potentials into the measurable range for the comparator. A current source is turned on to reduce 'vcurrent' to 'vcm', implementing the time duration $t_{\text {off }}$ as shown in Fig. 8. At the same time, the converter switch S (Fig. 5) is turned off. As soon as zero voltage difference $\Delta \mathrm{Vc}=0$ is detected between ' $\mathrm{vcm}$ ' and 'vcurrent' nodes, the switch is turned on again and the current source is turned off. With the chosen capacitor values a current of $70 \mathrm{nA}$ is necessary. The circuits in Fig. 7 represent a low-power solution to receive $\Delta \mathrm{Vc}$ without using a power consuming operational amplifier. The power consumption of this component is determined by the current source, which is in the low $\mathrm{nW}$ range.
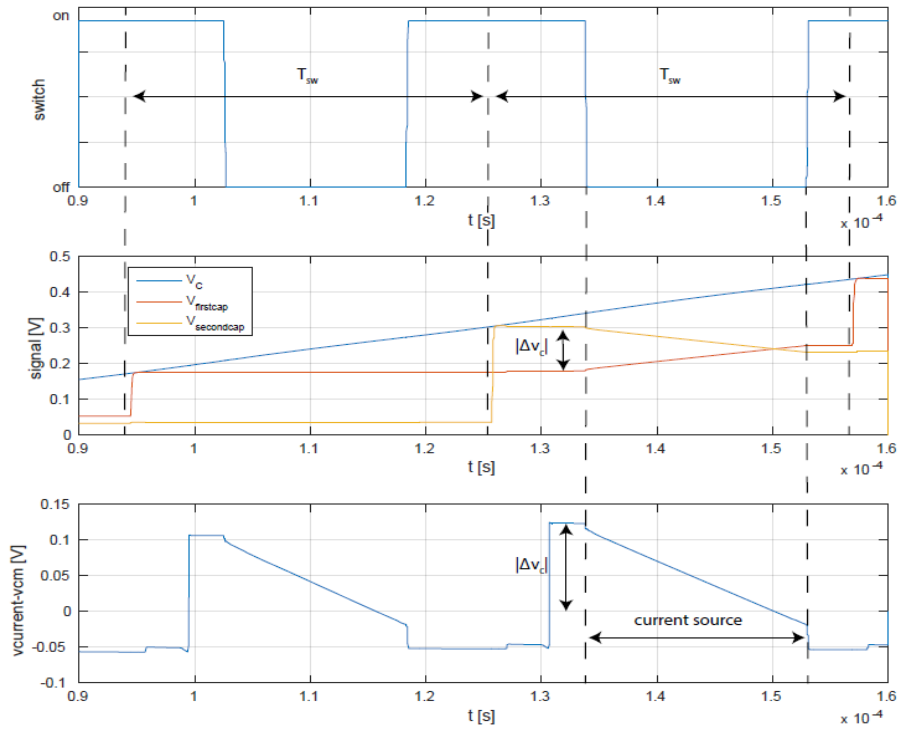

Fig. 8 Waveforms of switching cycle.

\section{E. Control FSM}

In order to operate the converter a control finite state machine (FSM) with nine states was developed as shown in Fig. 9. The inputs are the four comparator (Fig. 5) signals, an external signal for enabling the four comparator signals (enable), an external reset, and a clock signal. The FSM controls the converter switch and the control path circuits. It changes its state according to the clock signal. But the comparator signals depend on the burst. If a comparator measures a higher (or lower) voltage with respect to an earlier measurement, the comparator responds faster (or slower) and the comparator signal arrives earlier (or later) than in a prior measurement. Thus, the comparator signals are asynchronous with respect to the clock. To synchronize these signals two flip-flops are connected to each comparator signal in cascade. Furthermore, for low-power consumption, when no burst is available, the clock is turned off and the current consumption of all comparators is reduced to $15 \mathrm{nA}$. As soon as the comparator detects a burst its output signal directly turns on the clock.

\section{EXPERIMENTAL/SIMULATION RESULTS}

The converter chip is designed in a SMIC 130nm 1P8M CMOS technology and is shown Fig. 5. The design has been 
evaluated in simulations. The converter is analysed concerning its quiescent current and energy conversion efficiency per burst to charge a Li-Ion battery (Vo in Fig. 5) with a typical voltage ranging in $3.5 \mathrm{~V}-4.2 \mathrm{~V}$. The consumption of the converter yields a quiescent current of $250 \mathrm{nA}$ for no burst and a current of $3.2 \mathrm{uA}$ for a burst of $100 \mathrm{~ms}$. This results in an overall efficiency of 87\%-88\% with respect to the MPP for conversion of the AC output of the Kinetron micro-generator into usable power on the battery. Unlike other works, it is worth to mention that these values account for both internal losses and for MPPT accuracy. Moreover our design performs better than state of the art solutions in terms of conversion efficiency and quiescent current. The efficiency is referred to the power available from the transducer in the MPP $\mathrm{P}_{\text {inMax }}$ (i.e. $\mathrm{P}_{\text {in }}$ at the matched load) and evaluated as $\mathrm{P}_{\text {out }} / \mathrm{P}_{\text {inMax }} \times 100$.

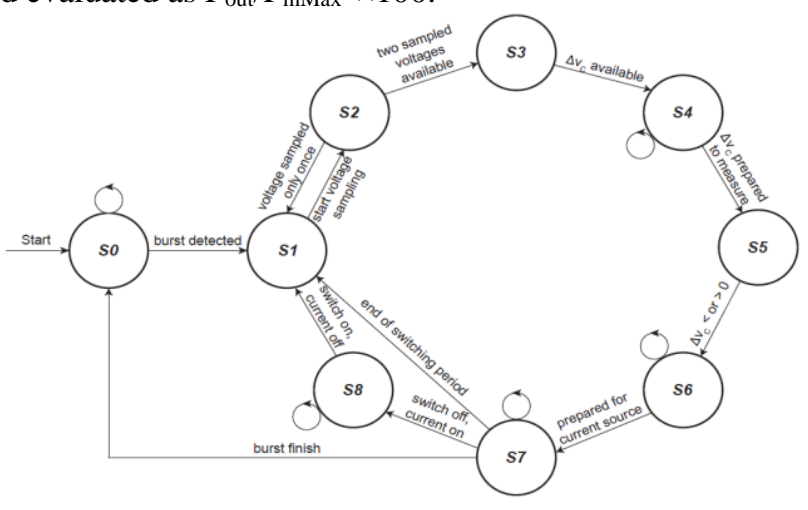

Fig. 9 State diagram of the control logic

Table I shows a comparison, in terms of quiescent current, peak efficiency, average efficiency and MPPT implementation, between the proposed solution, state of the art research papers ([21][30][31]) and two commercial products from Linear and Texas Instruments, which was coupled with a discrete NVC used as AC/DC converter in series with the kinetic generator. The micro-generator MGS 26.4[7] has been used as input transducer for the energy harvesting circuits. We accurately characterized the MGS 26.4 in our lab to acquire measured data for our circuit simulations. For the two commercial ICs (BQ25570 and Linear LTC3331) the whole system including the micro kinetic generator MGS 26.4 has been tested in the field. On the other hand, the data for [21][30][31] come as reported by the published works. It can be noticed from the table that our solution achieves the lowest quiescent current and highest average efficiency.

TABLE 1: COMPARISON WITH OTHER SOA EH CIRCUITS.

\begin{tabular}{|c|c|c|c|c|}
\hline Reference & Type & $\begin{array}{c}\text { Quiescent } \\
\text { Current }\end{array}$ & $\begin{array}{c}\text { Peak/avg } \\
\text { n[\%] }\end{array}$ & MPPT \\
\hline Custom IC [21]] & Resonant ac-dc & ND & $86.4 / \mathrm{NA}$ & $\mathrm{Y}$ \\
\hline Custom IC [30] & Active Full Bridge & $\mathrm{mW}$ & $81 / \mathrm{NA}$ & $\mathrm{Y}$ \\
\hline Custom IC [31] & Full-Wave Active Rect. & $10 \mu \mathrm{W}$ & $80-90 / \mathrm{NA}$ & $\mathrm{N}$ \\
\hline Discrete + BQ25570] & NVC+Boost Conv. & $500 \mathrm{nA}$ & $89 / 80$ & $\mathrm{Y}$ \\
\hline Linear LTC3331 & Full-Bridge Rectifier & $900 \mathrm{nA}$ & $50 / 30$ & $\mathrm{~N}$ \\
\hline THIS WORK & NVC+Control Circuit & $\mathbf{2 5 0 n A}$ & $\mathbf{8 9 / 8 6}$ & $\mathrm{Y}$ \\
\hline
\end{tabular}

\section{Conclusions}

An energy efficient converter has been designed for kinetic energy harvesting. Circuit characterization through simulations shows a quiescent current of only $250 \mathrm{nA}$, and the capability of maximizing both conversion efficiency and MPPT accuracy by transferring on the output up to $88 \%$ of the maximum available power with a matched load. The integrated circuits can be used for wearable kinetic harvesting supplying sensors devices. The chip layout and fabrication process is ongoing.

\section{REFERENCES}

[1] P. Spies, M. Pollak, L. Mateu, eds. Handbook of Energy Harvesting Power Supplies and Applications. CRC Press, 2015.

[2] Z. Wang, V. Leonov, P. Fiorini, C. V. Hoof, "Realization of a wearable miniaturized thermoelectric generator for human body applications," Sensors and Actuators A: Physical, 156(1), pp. 95-102, 2009.

[3] G. Snyder, "Thermoelectric energy harvesting," in Energy Harvesting Technologies, S. Priya and D. Inman, Eds., Springer 2009, pp. 325-336.

[4] M. Billinghurst, D. Busse "Rapid Prototyping for Wearables: Concept Design and Development for head-and wrist-mounted Wearables (Smart Watches and Google Glass)", Proceedings of the $9^{\text {th }}$ Int.l Conference on Tangible, Embedded, and Embodied Interaction. ACM, 2015.

[5] Gorlatova, M., Sarik, J., Grebla, G., Cong, M., Kymissis, I., \& Zussman, G. (2015). Movers and shakers: Kinetic energy harvesting for the internet of things. IEEE Journal on Selected Areas in Communications, 33(8), 1624-1639..

[6] M. Lossec, B. Multon, and H. Ben Ahmed, "Micro-kinetic generator: Modeling, energy conversion optimization and design considerations," in Melecon 2010 - 2010 15th IEEE Mediterranean Electrotechnical Conference, 2010, pp. 1516-1521

[7] "Kinetron MSG 26.4 Datasheet," 2016. [Online]. Available: http://www.kinetron.eu/wp-content/uploads/2015/01/MGS-26.4.pdf.

[8] Chen, Shin-Hao, et al. "A Direct AC-DC and DC-DC Cross-Source Energy Harvesting Circuit with Analog Iterating-Based MPPT Technique with $72.5 \%$ Conversion Efficiency and 94.6\% Tracking Efficiency." IEEE Transactions on Power Electronics 31.8 (2016): 5885-5899.

[9] . Chu, Yen-Chia, Dariusz Czarkowski, Jialin Zou, H. Jonathan Chao, Le-Ren ChangChien, Chih-Hsiang Chang, and N. Sertac Artan. "On-chip AC-DC multiple-powersupplies module for transcutaneously powered wearable medical devices." In Industry Applications Society Annual Meeting, 2016 IEEE, pp. 1-10. IEEE, 2016.

[10] Wahbah, Maisam, Mohammad Alhawari, Baker Mohammad, Hani Saleh, and Mohammed Ismail. "An AC-DC converter for human body-based vibration energy harvesting." Microelectronics Journal 55 (2016): 1-7.

[11] de Carli, Lucas G., Yuri Juppa, Adilson J. Cardoso, Carlos Galup-Montoro, and Márcio C. Schneider. "Maximizing the power conversion efficiency of ultra-lowvoltage CMOS multi-stage rectifiers." IEEE Tra) nsactions on Circuits and Systems I: Regular Papers 62, no. 4 (2015): 967-975.

[12] C. Peters, J. Handwerker, D. Maurath, and Y. Manoli, "An Ultra-Low-Voltage Active Rectifier for Energy Harvesting Applications," Circuits and Systems (ISCAS), Proceedings of 2010 IEEE, pp. 889-892, 2010

[13] Billinghurst, Mark, and Daniela Busse. "Rapid Prototyping for Wearables: Concept Design and Development for head-and wrist-mounted Wearables (Smart Watches and Google Glass)." Proceedings of the Ninth International Conference on Tangible, Embedded, and Embodied Interaction. ACM, 2015.

[14] A. S. Weddell, M. Magno, G. V. Merrett, D. Brunelli, B. M. Al-Hashimi and L. Benini, "A Survey of Multi-Source Energy Harvesting Systems," in Design, Automation \& Test in Europe Conference \& Exhibition (DATE), 2013, pp.905-910, March 2013

[15] Spadaro, L., Magno, M. and Benini, L., 2016, April. KinetiSee-A Perpetual Wearable Camera Acquisition System with a Kinetic Harvester. In 2016 15th ACM/IEEE International Conference on Information Processing in Sensor Networks (IPSN) (pp. 1-2). IEEE.

[16] Mauriello, Matthew, Michael Gubbels, and Jon E. Froehlich. "Social fabric fitness: the design and evaluation of wearable E-textile displays to support group running." Proceedings of the 32nd annual ACM conference on Human factors in computing systems. ACM, 2014

[17] Magno, M.; Boyle, D.; Brunelli, D.; O'Flynn, B.; Popovici, E.; Benini, L., "Extended Wireless Monitoring Through Intelligent Hybrid Energy Supply," Industrial Electronics, IEEE Transactions on , vol.61, no.4, pp.1871,1881, April 2014

[18] Brogan, Q.; O'Connor, T.; Dong Sam Ha, "Solar and thermal energy harvesting with a wearable jacket," Circuits and Systems (ISCAS), 2014 IEEE International Symposium on , vol., no., pp.1412,1415, 1-5 June 2014

[19] Wahbah, M.; Alhawari, M.; Mohammad, B.; Saleh, H.; Ismail, M., "Characterization of Human Body-Based Thermal and Vibration Energy Harvesting for Wearable Devices," Emerging and Selected Topics in Circuits and Systems, IEEE Journal on vol.4, no.3, pp.354,363, Sept. 2014

[20] Kwon, D., \& Rincón-Mora, G. A. (2010). A 2-\$ mu \$ m BiCMOS Rectifier-Free AC-DC Piezoelectric Energy Harvester-Charger IC. IEEE transactions on biomedical circuits and systems, 4(6), 400-409. 
[21] Y. Tang and A. Khaligh, "A Multiinput Bridgeless Resonant AC-DC Converter for Electromagnetic Energy Harvesting," in IEEE Transactions on Power Electronics, vol. 31, no. 3, pp. 2254-2263, March 2016

[22] Wang, Yu, Na Yan, Hao Min, and C-J. Shi. "A High-Efficiency Split-Merge Charge Pump for Solar Energy Harvesting." IEEE Transactions on Circuits and Systems II: Express Briefs (2016).

[23] Das, A., Gao, Y., \& Kim, T. T. H. (2016). A 220-mV Power-on-Reset Based SelfStarter With 2-nW Quiescent Power for Thermoelectric Energy Harvesting Systems. IEEE Transactions on Circuits and Systems I: Regular Papers.

[24] Lu, Yan, Haojuan Dai, Mo Huang, Man-Kay Law, Sai-Weng Sin, U. Seng-Pan, and Rui P. Martins. "A Wide Input Range Dual-Path CMOS Rectifier for RF Energy Harvesting." IEEE Transactions on Circuits and Systems II: Express Briefs (2016).

[25] M. Dini, A. Romani, M. Filippi, V. Bottarel, G. Ricotti, and M. Tartagni, "A Nanocurrent Power Management IC for Multiple Heterogeneous Energy Harvesting Sources," IEEE Trans. Power Electron., vol. 30, no. 10, pp. 5665-5680, 2015

[26] Fuketa, H., Shin-ichi, O., \& Matsukawa, T. (2016). Fully Integrated, 100-mV Minimum Input Voltage Converter with Gate-Boosted Charge Pump Kick-Started by LC Oscillator For Energy Harvesting. IEEE Transactions on Circuits and Systems II: Express Briefs.

[27] Wu, Liao, Xuan-Dien Do, Sang-Gug Lee, and Dong Sam Ha. "A Self-Powered and Optimal SSHI Circuit Integrated With an Active Rectifier for Piezoelectric Energy Harvesting." IEEE Transactions on Circuits and Systems I: Regular Papers (2016).

[28] M. Dini, A. Romani, M. Filippi, and M. Tartagni, "A Nanopower Synchronous Charge Extractor IC for Low-Voltage Piezoelectric Energy Harvesting With Residual Charge Inversion," IEEE Trans. Power Electron., vol. 31, no. 2, pp. 1263 1274, Feb. 2016.

[29] E. Dallago, A. Danioni, M. Marchesi, V. Nucita, and G. Venchi, "A Self-Powered Electronic Interface for Electromagnetic Energy Harvester," IEEE Trans. Power Electron., vol. 26, no. 11, pp. 3174-3182, Nov. 2011.

[30] Rahimi, Arian, et al. "Fully self-powered electromagnetic energy harvesting system with highly efficient dual rail output." IEEE Sensors Journal 12.6 (2012): 22872298.

[31] D. Maurath, P. F. Becker, D. Spreemann, and Y. Manoli, "Efficient Energy Harvesting With Electromagnetic Energy Transducers Using Active Low-Voltage Rectification and Maximum Power Point Tracking," IEEE J. Solid-State Circuits, vol. 47, no. 6, pp. 1369-1380, Jun. 2012. 